\title{
EFICIÊNCIA DOS SURFATANTES DE USO AGRICOLA NA REDUÇÃO DA TENSÃO SUPERFICIAL
}

\author{
Gilmar A. Montório ${ }^{1}$, Edivaldo D. Velini ${ }^{2}$, Cleber D. G. Maciel $^{3}$, Thiago Montório ${ }^{4}$ \\ ${ }^{1}$ Eng $^{\mathrm{o}}$ Agrônomo, Dr., Professor ESAPP, CP 88, Paraguaçu Paulista/SP. montorio@ @etonne.com.br \\ ${ }^{2}$ Eng $^{\mathrm{o}}$ Agrônomo, Dr., Professor FCA/ UNESP, Botucatu/ SP \\ ${ }^{3}$ Estudante curso Pós Graduação em Agronomia. FCA/ UNESP, Botucatu/ SP \\ ${ }^{4}$ Acadêmico de Agronomia. Escola Superior de Agronomia de Paraguaçu Paulista - ESAPP
}

\section{RESUMO}

O presente trabalho teve como objetivo estudar o comportamento da tensão superficial em gotas de solução elaboradas com diferentes concentrações de surfatantes. Para analisar a tensão fez-se pesagens das gotas formadas na extremidade de uma bureta, com os seguintes tratamentos combinados de forma fatorial (15x12) 15 surfatantes (Ag-Bem, Agral, Aterbane BR, Agrex, Break Thru, Extravon, Energic, Fixade, Gotafix, Haiten, Herbitensil, Iharaguem, Lanzar, Silwet L-77 e Wil Fix) e 12 concentrações $(0 ; 0,001 ; 0,0025 ; 0,005 ; 0,01 ; 0,025 ; 0,05 ; 0,1 ; 0,25 ; 0,5 \% ; 1$ e 2\%) num total de 180 tratamentos. A análise estatística dos dados foi realizada com auxilio do programa SAS e para análise de regressão, adotou-se o modelo de Mitscherlich ajustado e modificado para os dados obtidos.Observou-se que os surfatantes organosiliconados Break Thru e Silwet L-77 reduziram significativamente a tensão superficial $(19,87$ e 19,08 mN/m; respectivamente) e apresentaram os mais elevados coeficientes de eficácia (169,81 e 143,43). Wil Fix e Agrex apesar de terem apresentados baixos coeficientes de eficácia (1,52 e 4,01; respectivamente), reduziram significativamente a tensão superficial $(24,71$ e $29,29 \mathrm{mN} / \mathrm{m})$ porem numa concentração elevada próxima a $2 \%$ v/ v. Os demais surfatantes (Ag-Bem, Agral, Aterbane BR, Extravon, Energic, Fixade, Gotafix, Haiten, Herbitensil, Iharaguem e Lanzar) apresentaram tensões mínimas bastante próximas, portanto, a comparação entre eles pode ser feita exclusivamente pelo coeficiente de eficácia.

Palavras chaves: Surfatante, tensão superficial, método experimental, micelização. 


\title{
Study of the surface tension in drops of solutions with surfactants of agricultural use.
}

\begin{abstract}
The present work had the objective to study the behavior of the superficial tension in solution drops elaborated with different surfactants concentrations. To analyze the tension he/she made himself weight of the drops formed in the extremity of the burette, with the following combined treatments of form factorial (15x12) 15 surfactants (Ag-Bem, Agral, Aterbane BR, Agrex, Break Thru, Extravon, Energic, Fixade, Gotafix, Haiten, Herbitensil, Iharaguem, Lanzar, Silwet L-77 and Will Fix) and 12 concentrations $(0 ; 0,001 ; 0,0025 ; 0,005 ; 0,01 ; 0,025 ; 0,05 ; 0,1$; 0,$25 ; 0,5 \% ; 1$ and $2 \%$ ) in to total of 180 treatments. Analyze it statistics of the dates it was accomplished with I aid of the SAS program and it goes it analyzes of regression, the model of adjusted Mitscherlich was adopted and modified it goes the obtained it dates. It was observed that the surfactants organosilicons Break Thru and Silwet reduced the surface tension significantly $(19,87$ and $19,08 \mathrm{mN} / \mathrm{m}$; respectively) and they presented the highest coefficients of effectiveness $(169,81$ and 143,43). Will Fix and Agrex in spite of they have presented low coefficients of effectiveness (1,52 and 4,01; respectively), they reduced the superficial tension significantly $(24,71$ and $29,29 \mathrm{mN} / \mathrm{m})$ they put in the close high concentration attn $2 \% \mathrm{v} / \mathrm{v}$. The other surfactants presented quit close minimum tensions; the comparison among them can be made exclusively by the coefficient of effectiveness.
\end{abstract}

Key words: Surfactant, surface tension, experimental method, micelization.

\section{INTRODUÇÃO}

A água é um dissolvente universal para moléculas polarizadas e o veículo mais importante para diluir formulações de defensivos agrícolas aplicados através de pulverizações. Entretanto ela 
apresenta baixa capacidade de retenção quando aplicada sobre alvos com superfícies cerosas e hidrofóbicas (Stevens et al. 1993). Herbicidas aplicados na superfície foliar devem penetrar a cutícula e o plasmalema antes de atingir o citoplasma e ser posteriormente carreado para seu sítio de ação (Holoway \& Edgerton ,1992). A natureza hidrofóbica da cutícula age como barreira entre a folha e o ambiente, prevenindo a perda excessiva de água pela transpiração celular e protegendo a célula contra estresse biótico e abiótico. Variação na composição química, características morfológicas e estruturais da cera epicuticular entre espécies de plantas daninhas, associado às condições ambientais, são fatores determinantes da seletividade e eficácia de alguns herbicidas (Bacher \& Chamel, 1990; Newsom et al.1993). O controle eficiente de plantas daninhas por herbicidas aplicados na folhagem é dependente de sua translocação nas plantas. A velocidade de absorção e translocação é determinada por inúmeros fatores. Em alguns casos, a incidência de chuvas após a aplicação do herbicida pós-emergente pode comprometer a eficácia do produto. Também a eficiência de herbicidas pós-emergentes pode ser influenciada por diversos fatores: espécies e tamanho de plantas daninhas, condições ambientais, momento da aplicação, dose de aplicação, interação com outros herbicidas e uso de adjuvantes (Bridges, 1989; York et al., 1990). Adjuvantes são acrescentados à calda de pulverização com o objetivo de melhorar a eficiência das pulverizações foliares de herbicidas, reduzir o impacto das interferências ambientais e permitir uma penetração cuticular mais eficiente (Stougaard, 1997), facilitar o molhamento em superfícies hidrorepelentes e facilitar o contato da calda com a cutícula em superfície pilosas que tendem a manter as gotas suspensas (Kismann, 1996). Dentre esses, tipicamente os surfatantes reduzem a tensão superficial do líquido de pulverização, diminuindo o ângulo de contato (que depende da cerosidade da superfície e tensão superficial do líquido) das gotas isoladas sobre a superfície foliar, fazendo com que elas deixem de ser esféricas (Sing \& Mack, 1993; McWorther \& Ouzts, 1994). O acréscimo de surfatantes nas caldas aquosas de pulverização contribui para a 
formação de filmes líquidos sobre as superfícies foliares graças ao processo de coalescência das gotas. Diante do exposto, já faz parte das recomendações de herbicidas quando aplicados na modalidade pós-emergência, acrescentar surfatantes à calda de pulverização para usufruir os benefícios citados anteriormente, corroborando para a maior eficiência do produto. Os maiores agravantes são a falta de informação e a existência de poucos métodos experimentais que nos permita avaliar as propriedades físico-químicas dos surfatantes quanto a redução da tensão superficial, índice de eficiência, etc..

Logo esse trabalho teve como objetivo avaliar em diferentes concentrações, a mínima tensão superficial e o índice de eficiência apresentado pelos principais espalhantes adesionantes e umectantes utilizados no Brasil.

\section{MATERIAL E MÉTODOS}

As avaliações da tensão superficial foram realizadas no Laboratório de Nutrição da Escola Superior de Agronomia de Paraguaçu Paulista - ESAPP, em Paraguaçu Paulista-SP. Os tratamentos foram constituídos de 15 produtos adjuvantes comercializados no mercado Brasileiro (Quadro 1) e nas seguintes concentrações: 2\%; $1 \% ; 0,5 \% ; 0,25 \% ; 0,1 \% ; 0,05 \% ; 0,025 \% ; 0,01 \%$; 0,005\%; 0,0025\%; 0,001 e 0\% v/ v (água destilada). Utilizou-se uma balança de precisão em gramas com quatro casas decimais, óleo de soja, copos de Becker, balões volumétricos $(100 \mathrm{~mL}$ e $50 \mathrm{~mL}$ ) e bureta de $50 \mathrm{~mL}$.

A escolha das concentrações dos espalhantes foi feita a partir de recomendações dos fabricantes e dos resultados obtidos por Velini et al. (1993). Posteriormente estimou-se a tensão superficial dos tratamentos quantificando-se o peso das gotas formadas na extremidade da bureta em balança de precisão em gramas com quatro casas decimais, num tempo médio aproximado de 30 segundos. Inicialmente utilizou-se uma bureta calibrada com peso da água destilada. Para evitar perdas por evaporação usou-se uma camada de óleo de soja em um Becker de $25 \mathrm{~mL}$ 
colocados sobre a balança. Foram utilizadas 15 repetições. O capilar responsável pela formação da tensão superficial das gotas foi constituído pela extremidade da bureta, a qual para todos os tratamentos encontrava-se a uma altura de $5 \mathrm{~cm}$ acima da superfície do óleo, padronizando assim a interferência no caminhamento das gotas para todos os tratamentos durante o percurso de queda.

A calibração da bureta sempre foi acompanhada do tempo total de formação até a queda da gota na ponta da mesma, com auxílio de um cronômetro. Alem disso, à regulagem através de abertura e fechamento da torneira da bureta foram acompanhadas da altura da coluna de líquido, a qual foi mantida em $50 \mathrm{ml}$ na escala de graduação. Os dados de peso das gotas foram convertidos para tensão superficial, considerando uma média do peso de gotas da água destilada como 0,0726 $\mathrm{N} / \mathrm{m}$ (Newtons/metros), conforme metodologia desenvolvida por Costa et al. (1997).

A análise estatística dos dados foi realizada com auxílio do programa SAS e para análise de regressão dos mesmos, adotou-se o modelo representado pela equação de Mitscherlich. Para que o modelo de Mitscherlich se ajustasse aos dados obtidos, houve a necessidade de modifica-lo. Modelo original: $\quad \mathrm{Y}=\mathrm{A} \cdot\left[1-10^{-\mathrm{C} .}(\mathrm{X}+\mathrm{B})\right]$; Modelo utilizado: $\quad \mathrm{Y}=\mathrm{T}_{\text {água }}-\mathrm{A} \cdot\left[1-10^{-\mathrm{C} .(\mathrm{X}+\mathrm{B})}\right]$; Onde: $\mathrm{Y}=$ Tensão superficial, em $\mathrm{mN} / \mathrm{m} ; \mathrm{T}_{\text {água }}-\mathrm{A}=$ corresponde a mínima tensão superficial que pode ser alcançada com o surfatante; $\mathrm{T}_{\text {água }}=0,0726 \mathrm{~N} / \mathrm{m} ; \mathrm{C}=$ corresponde a eficiência do surfatante obtida diretamente da equação do modelo modificado; $X=$ Concentração do surfactante expresso em porcentagem e B = indica quanto do surfatante tem-se que colocar para se obter a mesma redução de tensão condicionada pela adição do herbicida na concentração considerada. Como não foram utilizados herbicidas, tal parâmetro recebeu valor igual a zero.

\section{RESULTADOS E DISCUSSÃO}

Os valores do coeficiente de eficácia (C) e de tensões superficiais $(\mathrm{mN} / \mathrm{m})$ mínimas alcançadas com os surfatantes estudados encontra-se no Quadro 2. No Quadro 3, estão as concentrações estimadas (\%) dos surfatantes que permitiram alcançar as tensões superficiais de 
$70,60,50,40,35,30,25$ e $20 \mathrm{mN} / \mathrm{m}$. Verifica-se que somente os surfatantes organosiliconados Silwet L-77 e Break Thru conseguiram atingir a tensão superficial de $20 \mathrm{mN} / \mathrm{m}$. Tal comportamento também é verificado na concentração de $25 \mathrm{mN} / \mathrm{m}$, juntamente com espalhante Will Fix. Apresentando nível de $30 \mathrm{mN} / \mathrm{m}$, alem dos organosiliconados e Will Fix tem os seguintes surfatantes: Fixade, Herbitensil, Iharaguem, Haiten e Agrex. Todos os produtos permitiram alcançar a tensão de $35 \mathrm{mN} / \mathrm{m}$. A partir dessa tensão, verifica-se que os redutores de tensão mantêm o mesmo comportamento e a mesma relação entre si, podendo ser agrupados em ordens decrescentes da seguinte forma: Break Thru com Silwet L-77, Fixade com Herbitensil e depois Aterbane com Iharaguem. Com resultados inferiores e numa faixa mais ampla tem-se: Energic, Agral, Gotafix e Haiten. Por último, observa-se Ag-Bem, Lanzar, Agrex e Wil Fix reduzindo a tensão superficial em concentrações maiores que os anteriores. Por exemplo, o surfatante Wil Fix reduziu a tensão superficial da solução a níveis de $35 \mathrm{mN} / \mathrm{m}$ numa concentração 125 vezes superior aos organosiliconados e aproximadamente 38 vezes superior ao Fixade e Herbitensil. Tal comportamento também é observado para o surfatante Agrex (ambos apresentam na sua composição ácido dodecil benzeno sulfônico). Portanto, diante dessa condição alem de observar a eficácia na redução da tensão, deve-se também levar em consideração o custo benefício para tais tratamentos. Na Figura 1, encontram-se as curvas de tensão superficial de todos os surfatantes utilizados no estudo, concentrando-os num só gráfico.

O maior número de trabalhos encontrados na literatura referem-se a mistura de surfatantes com os diferentes tipos de herbicidas e na maioria das vezes considerando o grau de tensão superficial como critério de avaliação. Realizando estudos com espalhantes adesionantes isoladamente e em diferentes concentrações tem-se o trabalho de Matuo et al. (1989), Mendonça et al. (2000), Costa (1997) e Velini (2000). A exceção de Matuo et al. (1989) que utilizaram um tensiômetro Krus modelo K10 para avaliar a tensão superficial, os demais autores se utilizaram de uma bureta previamente calibrada onde a gota formada na extremidade capilar da mesma foi pesada. Matuo et al. (1989) quando estudaram os efeitos de alguns adjuvantes nas propriedades físicas do líquido obtiveram resultados que estão de acordo com aqueles observados nesse trabalho, utilizando bureta previamente calibrada. Para o espalhante Aterbane, Mendonça et al. (1996) e Costa (1997) encontraram a mínima tensão superficial igual a 32,44 e 32,45, respectivamente. Aterbane nesse estudo apresentou mínima tensão superficial igual a 32,68 $\mathrm{mN} / \mathrm{m}$. Esses mesmos autores encontraram um coeficiente de eficácia igual a 44,46 e 46,09; respectivamente, também de acordo com os resultados obtidos nesse estudo. $\mathrm{O}$ espalhante 
adesionante Extravon apresentou mínima tesão superficial igual a 30,4679 mN/m com um coeficiente de eficácia de 30,3853. Quando Mendonça et al. (1999) e Costa (1997) estudaram esse surfatante, encontraram a mínima tensão superficial próxima a $31,0 \mathrm{mN} / \mathrm{m}$, de acordo com os resultados obtidos nessa pesquisa. Esses mesmos autores, também estudando os efeitos de surfatantes sobre a tensão superficial em soluções de glyphosate e rodeo, encontraram os valores da mínima tensão superficial para o siliconado Silwet L-77 próximos a $19 \mathrm{mN} / \mathrm{m}$. Stevens et al. (1993) quando estudaram a adesão de gotas de pulverização e a tensão superficial dinâmica comparando surfatante siliconados com os surfatantes convencionais, observaram que todos os organo-siliconados testados apresentaram tensão de equilíbrio com valores próximos a $20 \mathrm{mN} / \mathrm{m}$ na concentração de $0,05 \%$ v/ v. Os espalhantes não siliconados convencionais mostraram níveis de equilíbrio próximos a $30,3 \mathrm{mN} / \mathrm{m}$ com níveis de concentrações maiores que às observadas nos surfatantes organosiliconados (Buick et al., 1993; Singh, 1993).

Como os surfatantes siliconados apresentam conformação molecular de forma mais maleável devido à ligação carbono-silício, este propicia condição para que o grupamento hidrófobo do surfatante seja mais paralelo à superfície da gota, do que o grupamento hidrófilo que se estende mais para o interior da mesma. Essa confirmação produz redução da tensão superficial mais rapidamente e a valores menores da tensão superficial do que aqueles observados para os surfatantes não organo-siliconados que evidenciam conformação mais rígida, devido às ligações carbono-carbono (Costa, 1997). Considerando as informações apresentadas, o surfatante ideal é o que apresenta elevado coeficiente de eficácia associada às baixas tensões mínimas. Os surfatantes organosiliconados combinam estas características ideais. Outra exceção é o Wil Fix com tensões mínimas de $24,7 \mathrm{mN} / \mathrm{m}$, superior apenas à dos organosiliconados, mas que apresentou coeficiente de eficácia extremamente baixo.Como os demais surfatantes apresentaram tensões mínimas bastante próximas, a comparação entre eles pode ser feita exclusivamente pelo coeficiente de eficácia. Em termos de classificação dos surfatantes quanto à eficiência, o único produto que se adequa ao padrão geral é o Will Fix. Embora a tensão mínima alcançada o coloque como o terceiro melhor produto, após os organosiliconados, observa-se que este surfatante foi o que exigiu maiores concentrações para alcançar a mínima tensão superficial $(24,7$ $\mathrm{mN} / \mathrm{m}$ a $2 \%)$.

O Agrex, o segundo menos eficiente, foi superior ao Wil Fix em termos de coeficiente de eficácia, mas apresentou tensão mínima superior à proporcionada pelo mesmo. Os produtos Lanzar e Ag-Bem, incluídos entre os menos eficientes $\left(12^{\circ}\right.$ e $\left.13^{\circ}\right)$ apresentam formulações do tipo 
concentrado emulsionável. Contudo o Ag-Bem tem como principal finalidade à adesão foliar e para isso apresenta em sua composição resinas que se polimerizam em contato com o ar. Este não é o caso do Lanzar considerado, também, como um dos surfatantes menos eficientes em termos de redução de tensão.

Os produtos não organosiliconados Fixade, Herbitensil, Aterbane e Energic apresentaram coeficiente de eficácia elevado. Tal comportamento se deve certamente por se tratar de surfatantes que apresentam as maiores concentrações de ingrediente ativo/litro. Pelo Quadro 1, observa-se que tais produtos apresentam as seguintes concentrações: 900 g/ 1, 526 g/ 1, 466 g/ 1 e 452 g/ 1; respectivamente. A exceção dos organosiliconados, o Wil Fix, Agrex, Ag-Bem e Lanzar, os demais surfatantes apresentam concentrações menores que $250 \mathrm{~g} / 1$.

\section{CONCLUSÕES}

Nas condições em que o estudo foi desenvolvido, pode-se chegar as seguintes conclusões:

Os surfatantes siliconados Silwet L-77 e Break Thru mostraram serem os mais eficientes dentre aqueles testados para redução da tensão superficial, alcançando as menores tensões mínimas e os maiores coeficientes de eficácia iguais;

Os surfatantes não siliconados Fixade, Herbitensil, Iharaguem, Extravon, Energic, Agral, Gotafix, Haiten e Aterbane mostraram serem os mais eficientes dentre aqueles testados para redução da tensão superficial, alcançando as menores tensões mínimas e os maiores coeficientes de eficácia;

Os surfatantes não siliconados Lanzar e Ag-Bem apresentaram comportamentos intermediário. Já os surfatantes Will Fix e Agrex foram os menos eficientes, apresentando baixos coeficientes de eficácia.

\section{LITERATURA CONSULTADA}

BACKER, E.A., CHAMEL, A.R. Herbicide penetration across isolated and intact leaf cuticles. Pesticide Science, v. 29, p.187-96, 1990. 
BRIDGES, D.C. Adjuvant and $\mathrm{pH}$ effects on sethoxydim and cletodim activity on rhizome johnsongrass (Sorghum halepense). Weed Technology, v.3, p.615-20, 1989.

BUICK, R.D., BUCHAN, G.D., FIELD, R. J. The role of surface tension of spreading droplets in absorption of a herbicide formulation via leaf stomata. Pesticide Science, v. 38, p.227-35, 1993.

COSTA, E.A.D. Efeitos de surfatantes sobre a tensão superficial de soluções de rodeo. Botucatu, 1997. 72p. Dissertação (mestrado em Proteção de Plantas)-Faculdade de Ciências Agronômicas, Universidade Estadual Paulista.

HOLLOWAY, P.J., EDGERTON, M. Effects of formulation with different adjuvants on foliar uptake of difenzoquat and 2,4-D. Weed Research, v. 32, p.183-95, 1992.

KISSMANN, K.G. Adjuvantes para caldas de defensivos agrícolas. BASF: São Paulo, 1996. $45 \mathrm{p}$.

MATUO, T., NAKAMURA, S.H., ALMEIDA, A. Efeitos de alguns adjuvantes da pulverização nas propriedades físicas do líquido. Summa Phytopathologica, v.15, p.163-173, 1989.

MCWHORTHER, C.G., OUZTS, R. Leaf surface morphology of Erythoxylum sp. and droplet spread. Weed Science, v.42, p.18-26, 1994.

MENDONÇA, G.G.; VELINI, E.D.; MARTINS, D.; MENDONÇA, C.G. Efeito de surfatantes sobre a tensão superficial e a área de molhamento de soluções de glyphosate sobre folhas de tiririca. Planta Daninha, v.17, p.355-365, 200.

MENDONÇA, C.G., VELINI, E.D., MARTINS, D., MENDONÇA, C.G. Efeito de surfatantes sobre a tensão superficial e a área de molhamento de soluções de ghyphosate sobre folhas de tiririca. Planta Daninha, v.17, p.355-65, 2000. 
NEWSOM, L. J., SHAW, D.R., HUBBARD, T.F. Absorption, translocation and metabolism of 263,222 in peanut (Arachis hipogaea), soybean (Glycine max) and selected weeds. Weed Science, v.41, p. 523-27, 1993.

SINGH, M., MACK, R.E. Effect of organosilicone-based adjuvants on herbicide efficacy. Pesticide Science, v.38, p.219-25, 1993.

STEVENS, P.J.G., KIMBERLEY, M.O., MURPHY, D.S. Adhesion of spray droplets to foliage: the role of dynamic surface tension and advantages of organosilicone surfactants. Pesticide Science, v.38, p. 237-45, 1993.

STOUGAARD, R.M. Adjuvant combinations with quizalofop for wild oat (Avena fatua) control in pepermit (Mentha piperita). Weed Technology, v.11, p.45-50, 1997.

VELINI, E.D., GAVLAK, R., RIBEIRO, O.C. Avaliação da tensão superficial de soluções do espalhante adesivo alquil fenol poliglicoléter, isolado ou em misturas com o herbicida glifosate. In.. CONGRESSO BRASILEIRO DE HERBICIDAS E PLANTAS DANINHAS, 19, 1993, Londrina. Resumos... Londrina: Sociedade Brasileira de Herbicidas e Plantas Daninhas, 1993. p.264.

YORK, A.C., JORDAN, D.L., WILCUT, J.W. Effects of (NH4)2SO4 and BCH 81508 S on efficacy of sethoxydim. Weed Technology, v.4, p76-80, 1990. 
Quadro 1. Caracterização dos surfatantes utilizados no experimento. Botucatu - SP, 2000.

\begin{tabular}{|c|c|c|c|c|}
\hline $\begin{array}{c}\text { Nome } \\
\text { Comercial }\end{array}$ & Nome do Ativo & $\begin{array}{c}\text { Concent. } \\
(\mathrm{g} / \mathrm{l})\end{array}$ & Característica & $\underset{*}{\text { Formul. }}$ \\
\hline Ag-Bem & $\begin{array}{l}\text { Resina sintética+agente tenso } \\
\text { ativo aniônico emulsionado }\end{array}$ & $387+129$ & Aniônico & $\mathrm{CE}$ \\
\hline Agral & Nonil fenol poli etanol (etilenoxi) & 200 & Não iônico & SAqc \\
\hline $\begin{array}{l}\text { Aterbane } \\
\text { BR }\end{array}$ & $\begin{array}{l}\text { Álcoofenóis+óxido de } \\
\text { eteno+sulfonatos orgânicos }\end{array}$ & 466 & Não iônico & SAq \\
\hline Agrex & Ácido dodecilbenzeno sulfônico & 50 & Iônico & SAqc \\
\hline Break Thru & Poliéter polimetil siloxano & 750 & Não iônico & CS \\
\hline Extravon & Alquil fenol poliglicol éter & 250 & Não ionico & SAqc \\
\hline Energic & $\begin{array}{c}\text { Nonil fenoxipoli etanol } \\
\text { (etilenoxi)+sal sódico de ácido } \\
\text { dodecilbenzeno sulfônico }\end{array}$ & $226+226$ & Não iônico / Iônico & SNAqc \\
\hline Fixade & Nonil fenol etoxilado & 900 & Não iônico & SNAqc \\
\hline Gotafix & Nonil fenol polietileno glicol éter & 125 & Não iônico & SAqc \\
\hline Haiten & Polioxietileno alquil fenol éter & 200 & Não iônico & SAqc \\
\hline Herbitensil & $\begin{array}{l}\text { Nonil fenol oxietilado+álcool } \\
\text { isopropilíco }\end{array}$ & $400+125$ & Não iônico & SAqc \\
\hline Ihraguem & Alquil fenol polioxietileno éter & 200 & Não iônico & SAqc \\
\hline Lanzar & Aquil éster etoxilado do $\mathrm{H}_{2} \mathrm{PO}_{4}$ & 280 & Não iônico & $\mathrm{CE}$ \\
\hline Silwet L-77 & $\begin{array}{l}\text { Polimetil siloxano } \\
\text { organomodificado }\end{array}$ & 990 & Não iônico & SNAqc \\
\hline Wil Fix & Ácido dodecilbenzeno sulfônico & 300 & Iônico & SAqc \\
\hline
\end{tabular}

* CE = Concentrado Emulsionável; SAqC = Solução aquosa concentrada; SNAqC = Solução não aquosa concentrada; $\mathrm{CS}=$ Concentrado solúvel 
Quadro 2. Resultados das análises de variância e regressão entre as tensões superficiais e as concentrações dos surfatantes (\%), utilizando o modelo de Mitscherlich modificado. ESAPP, Paraguaçu Paulista, SP, 2000

\begin{tabular}{|c|c|c|c|c|c|c|c|c|c|c|c|c|c|c|c|}
\hline \multirow[t]{2}{*}{ MODELO } & \multicolumn{15}{|c|}{$Y=72,6-A^{*}\left(1-10^{\wedge}(-C(B+X))\right)$} \\
\hline & Agral & Gotafix & Agrex & Ag-bem & Iharaguem & Energic & Aterbane & Fixade & Extravon & Lanzar & Wil fix & Haiten & Herbitensil & Silwet L-77 & $\begin{array}{c}\text { Break } \\
\text { Thru }\end{array}$ \\
\hline Estimativa & A 42,1353 & 41,6930 & 43,3112 & 39,8942 & 43,0451 & 41,8596 & 39,9188 & 44,3655 & 42,1321 & 40,1935 & 47,8916 & 44,6610 & 42,9372 & 53,5180 & 52,7292 \\
\hline dos & 0 & 0 & 0 & 0 & 0 & 0 & 0 & 0 & 0 & 0 & 0 & 0 & 0 & 0 & 0 \\
\hline Parâmetros & C 24,3076 & 22,6766 & 4,0134 & 11,2419 & 36,1074 & 27,8094 & 46,3822 & 89,2793 & 30,3853 & 8,6090 & 1,5170 & 21,3802 & 63,7398 & 142,4336 & 169,8138 \\
\hline Tensão mínima & 30,4647 & 30,9070 & 29,2888 & 32,7058 & 29,5549 & 30,7404 & 32,6812 & 28,2345 & 30,4679 & 32,4065 & 24,7527 & 27,9390 & 29,6628 & 19,0820 & 19,8707 \\
\hline S Q Regressão & 48279,0106 & 56444,5902 & 52688,2222 & 51628,0120 & 52986,5639 & 52108,0854 & 44010,5615 & 41486,4630 & 49279,188 & 44322,5057 & 59033,883 & 63197,67 & 37830,2099 & 72578,557 & 67690,426 \\
\hline S Q Resíduo & 419,1144 & 849,6219 & 1290,9129 & 169,6792 & 625,2649 & 339,1615 & 878,7622 & 206,5520 & 585,9187 & 889,7286 & 311,1685 & 459,1128 & 1232,8773 & 331,4248 & 252,4347 \\
\hline F Regressão** & 189014,13 & 402015,857 & 62620,02 & 358300,76 & 595035,20 & 1348090,66 & 624394,72 & 102979,68 & 1850039,5 & 179601,93 & 787994,80 & 216802,6 & 2386333,44 & 3872452,88 & 957269,57 \\
\hline $\mathrm{R}^{2}$ & 0,9905 & 0,9847 & 0,9735 & 0,9963 & 0,9881 & 0,9934 & 0,9802 & 0,9935 & 0,9882 & 0,9794 & 0,9945 & 0,9920 & 0,9684 & 0,9954 & 0,9961 \\
\hline
\end{tabular}

Resumo das análises de variâncias

\begin{tabular}{|c|c|c|c|c|c|c|c|c|c|c|c|c|c|c|c|}
\hline $\begin{array}{c}\text { S Q } \\
\text { Tratamentos }\end{array}$ & 48698,12 & 57294,21 & 53979,13 & 51797,69 & 53611,83 & 52447,25 & 44889,32 & 42053,01 & 49865,11 & 45212,23 & 59345,05 & 63656,78 & 39063,78 & 72909,98 & 67942,86 \\
\hline F tratamentos** & 17332,27 & 37097,01 & 5832,21 & 32679,85 & 54732,45 & 99999,99 & 57896,55 & 9408,00 & 99999,99 & 16655,20 & 72013,49 & 19852,51 & 99999,99 & 99999,99 & 80746,18 \\
\hline C.V. $(\%)$ & 1,1060 & 0,7937 & 1,7327 & 0,7442 & 0,6784 & 0,4287 & 0,5883 & 1,6403 & 0,3626 & 0,9753 & 0,4848 & 1,1869 & 0,3081 & 0,4216 & 0,8202 \\
\hline
\end{tabular}

**Todos os tratamentos foram significativos a $1 \%$ de probabilidade

$\mathrm{Y}=$ Tensão superficial $(\mathrm{mN} / \mathrm{m}) ; \mathrm{X}=$ concentração do adjuvante (\%); a, b e c são as estimativas dos parâmetros do modelo. 
Quadro 3. Coeficiente de eficácia e tensões mínimas alcançadas com o uso dos surfatantes estudados. Dados ordenados de forma crescente segundo os coeficientes de eficácia

\begin{tabular}{lccc}
\hline \multicolumn{1}{c}{ Surfatante } & “C” & $\begin{array}{c}\text { Tensão mínima } \\
(\mathbf{7 2 , 6 - \mathbf { A } )}\end{array}$ & $\begin{array}{c}\text { Classificação } \\
\text { Quanto a Eficácia }\end{array}$ \\
\hline Wil fix & 1,52 & 24,71 & 15 \\
Agrex & 4,01 & 29,29 & 14 \\
Lanzar & 8,61 & 32,41 & 13 \\
Ag-bem & 11,24 & 32,71 & 12 \\
Haiten & 21,38 & 27,94 & 11 \\
Gotafix & 22,68 & 30,91 & 10 \\
Agral & 24,31 & 30,46 & 9 \\
Energic & 27,81 & 30,74 & 8 \\
Extravon & 30,39 & 30,47 & 7 \\
Iharaguem & 36,11 & 29,55 & 6 \\
Aterbane & 46,38 & 32,68 & 5 \\
Herbitensil & 63,74 & 29,66 & 4 \\
Fixade & 89,28 & 28,23 & 3 \\
Silwet L-77 & 143,43 & 19,08 & 2
\end{tabular}

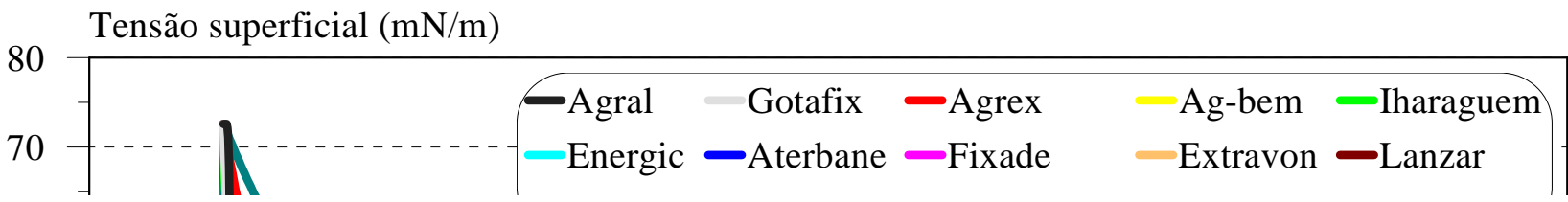



Quadro 4. Concentrações $(\%) \mathrm{v} / \mathrm{v}$ estimadas dos surfatantes relacionadas às tensões superficiais de 70, 60, 50, 40, 35, 30, 25 e 20 $\mathrm{mN} / \mathrm{m}$. Dados ordenados segundo concentrações estimadas decrescentes.

\begin{tabular}{|c|c|c|c|c|c|c|c|c|}
\hline \multirow{3}{*}{ Surfatante } & \multicolumn{8}{|c|}{ Tensões estimadas (mN/m) } \\
\hline & 70 & 60 & 50 & 40 & 35 & 30 & 25 & 20 \\
\hline & \multicolumn{8}{|c|}{$\% \quad \mathrm{v} / \mathrm{v}$} \\
\hline 1. Wil Fix & 0,01598 & 0,08740 & 0,18278 & 0,32683 & 0,44020 & 0,63063 & 1,46043 & - \\
\hline 2. Agrex & 0,00670 & 0,03720 & 0,07983 & 0,15118 & 0,21923 & 0,44466 & - & - \\
\hline 3. Lanzar & 0,00337 & 0,01897 & 0,04168 & 0,08406 & 0,13826 & - & - & - \\
\hline 4. Ag-Bem & 0,00260 & 0,01466 & 0,03229 & 0,06564 & 0,11033 & - & - & - \\
\hline 5. Haiten & 0,00122 & 0,00673 & 0,01433 & 0,02659 & 0,03747 & 0,06248 & - & - \\
\hline 6. Gotafix & 0,00123 & 0,00689 & 0,01496 & 0,02916 & 0,04445 & - & - & - \\
\hline 7. Agral & 0,00114 & 0,00635 & 0,01373 & 0,02655 & 0,03982 & - & - & - \\
\hline 8. Energic & 0,00100 & 0,00559 & 0,01212 & 0,02356 & 0,03569 & - & - & - \\
\hline 9. Extravon & 0,00091 & 0,00508 & 0,01099 & 0,02124 & 0,03187 & - & - & - \\
\hline 10. Iharaguen & 0,00075 & 0,00417 & 0,00895 & 0,01703 & 0,02487 & 0,05499 & - & - \\
\hline 11. Aterbane & 0,00063 & 0,00355 & 0,00782 & 0,01588 & 0,02665 & - & & - \\
\hline 12. Herbitensil & 0,00043 & 0,00237 & 0,00509 & 0,00970 & 0,01421 & 0,03302 & - & - \\
\hline 13. Fixade & 0,00029 & 0,00163 & 0,00346 & 0,00646 & 0,00915 & 0,01568 & - & - \\
\hline 14. Silwet L-77 & 0,00015 & 0,00081 & 0,00166 & 0,00284 & 0,00367 & 0,00481 & 0,00667 & 0,01231 \\
\hline 15. Break Thru & 0,00013 & 0,00070 & 0,00143 & 0,00246 & 0,00319 & 0,00422 & 0,00596 & 0,01537 \\
\hline
\end{tabular}

